\title{
SISTEMATIZAÇÃO DO PROJETO INFORMACIONAL DE UM ELETRODO DE RADIOFREQUÊNCIA
}

\section{SYSTEMATIZATION OF THE RADIO FREQUENCY ELECTRODE INFORMATIONAL DESIGN}

\author{
Beatriz Araujo Rodrigues* E-mail: beatrizaraujorodrigues@gmail.com \\ Andrea Cristina Santos* E-mail: andreasantos@unb.br \\ Viviane Vasconcellos Ferreira Grubisic* E-mail: vivianegrubisic@unb.br \\ *Universidade de Brasília (UnB), Brasília, DF
}

\begin{abstract}
Resumo: $O$ equipamento médico de rádio frequência (RFA) é utilizado como forma de tratamento do Carcinoma Hepatocelular (CHC). Segundo a Organização Mundial da Saúde (OMS), o CHC é a segunda causa de óbito por câncer no mundo. Dentre as formas de tratamento, existem procedimentos utilizando equipamentos de RFA minimamente invasivos capazes de extinguir tumores em órgãos vitais. Dentro desse contexto, o presente artigo tem como objetivo apresentar a fase de projeto informacional de um eletrodo de radiofrequência. A confiabilidade de um equipamento médico (EM) é um dos principais atributos exigidos por órgãos reguladores de um sistema de saúde, uma vez que o ambiente hospitalar apresenta características singulares. Assim, é feita uma revisão bibliográfica sobre confiabilidade e Processo de Projeto de Equipamentos Médicos (PPEM). Após, com base em uma metodologia de projeto foi possível identificar as necessidades e os requisitos dos clientes, os requisitos do projeto para então definir as especificações - meta do EM. O desenvolvimento do eletrodo de radiofrequência faz parte do projeto Software of Intensive Ablation (SOFIA), da Universidade de Brasília (UnB), financiado pelo Ministério da Saúde. O resultado da fase de projeto informacional, ou seja, as especificações meta servirão de base para o desenvolvimento das fases seguintes do projeto, logo após o resumo, três palavras-chave, as quais devem destacar de modo claro a temática do artigo.
\end{abstract}

Palavras-chave: Equipamento médico. Confiabilidade. Projeto informacional. Eletrodo de alta frequência.

\begin{abstract}
Medical radio frequency equipment (RFA) is used as a way of treating Hepatocellular Carcinoma (HCC). According to the World Health Organization (WHO), HCC is the second leading cause of cancer death worldwide. One treatment is the procedure using minimally invasive RFA equipment capable of extinguishing tumors in vital organs. Within this context, this article aims to present the informational design phase of a radiofrequency electrode. The reliability of medical equipment is one of the main attributes required by regulatory bodies of a health system, since the hospital environment has unique characteristics. Thus, a bibliographic review on reliability and Medical Equipment Design Process are made. Then, based on a design methodology, it was possible to identify the needs and requirements of the customers, the project requirements and then define the specifications - target of the medical equipment. The development of the radio frequency electrode is part of the Software of Intensive Ablation (SOFIA) project, of the Brasilia University (UnB), funded by the Health Ministry. The result of the informational design phase, that is, the specifications - target will serve as the basis for the following design phases.
\end{abstract}

Keywords: Medical equipment. Reliability. Informational design. Radiofrequency electrode. 


\section{INTRODUÇÃO}

O Brasil é um mercado em crescimento na área de dispositivos médicos e equipamentos, de acordo com dados do ShareScope - o serviço de tamanho/compartilhamento de mercado desenvolvido para as marcas de dispositivos médicos da LatAm pela Global Health Intelligence. A análise de dados de importação da ShareScope indica que o valor total de equipamentos e dispositivos médicos importados para o Brasil em 2017 foi superior a US 3,1 bilhões, um aumento de $15 \%$ em relação a 2016 , quando o valor dos equipamentos médicos (EM) importados para o Brasil caiu 14\% (ROMERO; CORPART, 2018).

O Brasil importou mais de 4,6 bilhões de unidades de equipamentos e dispositivos médicos em 2017. Inicialmente, esse número pode parecer alto, mas isso é porque ele não apenas considera equipamentos de capital como máquinas de ressonância magnética e tomografia computadorizada, scanners, mas também peças menores, como seringas, stents, peças e outros instrumentos que são encomendados em grandes quantidades (ROMERO; CORPART, 2018).

A funcionalidade de um EM é complexa, interconectada e interoperacional, devido a sua relação direta com a saúde e vida dos pacientes. Desta forma, as preocupações com a eficácia, segurança e confiabilidade crescem, uma vez que qualquer falha devido a razões diversas pode ser altamente dispendiosa em termos de lesões, fatalidades e perdas econômicas (DHILLON, 2000, MARTINI, 2005; CHENG; DAS; PECHT, 2011; LIN et al., 2014).

Para tal, a Agência Nacional de Vigilância Sanitária (Anvisa) exige que todos os dispositivos médicos sejam seguros e eficazes para o seu propósito por meio de um processo de registro que inclui certificado de testes de qualidade e boas práticas de fabricação, de forma a garantir confiabilidade durante a operação do serviço prestado.

Desenvolver uma especificação meta de projeto confiável consiste em um processo iterativo entre clientes, desenvolvedores e órgãos legisladores, por exemplo. Infelizmente, a maioria das empresas considera a confiabilidade somente quando o projeto do produto já está aprovado, não havendo tempo hábil para mudanças significativas no projeto (RAHEJA; GULLO, 2012; HEGDE; RAHEJA, 2010).

Revista Produção Online. Florianópolis, SC, v. 20, n. 4, p. 1238- 1259, 2020. 
Por possuir um caráter interconectado e simultâneo, a confiabilidade de um equipamento médico deve considerar também suas áreas correlatas como: segurança, avaliação de risco, qualidade, custos, manutenção e disponibilidade (DHILLON, 2000).

Submeter um equipamento médico a um programa de confiabilidade exige uma abordagem sistemática do seu processo de desenvolvimento a fim de garantir que os requisitos regulatórios sejam adequadamente satisfeitos. Para tal, é preciso entender claramente a função pretendida do produto e suas potenciais falhas. As funções pretendidas são funcionalidades que representam as necessidades dos clientes (que são todas as pessoas envolvidas no processo produtivo, inclusive os órgãos regulamentadores), representadas posteriormente por requisitos de clientes, requisitos de projeto e por fim, especificações - meta do produto (WOO, 2017).

Diante deste contexto, o presente artigo tem o objetivo de apresentar a fase de projeto informacional de um eletrodo de Radio Frequência desenvolvido no projeto Software of Intensive Ablation (SOFIA) da Universidade de Brasília (UnB) para ablação hepática. Tanto a fase de projeto conceitual, engenharia básica e projeto detalhado, próximas fases do desenvolvimento serão executadas buscando atender as especificações-meta definidas na fase de projeto informacional. Para tal, primeiramente será apresentado uma breve revisão bibliográfica sobre conceitos básicos relacionados a confiabilidade e PPEM. Em seguida, tem-se o estudo de caso e por fim, as considerações finais.

\section{CONFIABILIDADE}

Confiabilidade é definida como a probabilidade de um item, seja um componente ou equipamento de executar todas as funções por um tempo especificado e condições de uso especificadas. Por sua vez, disponibilidade: é a capacidade de um item, mediante manutenção apropriada, desempenhar sua função requerida em um determinado instante do tempo ou em um período de tempo predeterminado. Calculada pela probabilidade do equipamento estar operante quando necessitado. Por sua vez, mantenabilidade é a capacidade de um item ser mantido ou recolocado em condições de executar suas funções requeridas, mediante condições preestabelecidas de uso, quando submetido à manutenção sob 
condições predeterminadas e usando recursos e procedimentos padrão (ABNT NBR 5462: 1994).

As áreas associadas à confiabilidade de dispositivos médicos são aquelas que influenciam direta ou indiretamente o seu desenvolvimento e operação, sendo: qualidade, segurança, análise de risco, manutenção, fatores humanos/ usabilidade e custo (KING; FRIES; JOHNSON, 2014). O Quadro 1 apresenta a definição de cada uma delas.

Quadro 1 - Áreas associadas com a confiabilidade

\begin{tabular}{|l|l|}
\hline \multicolumn{1}{|c|}{$\begin{array}{c}\text { Áreas } \\
\text { associadas }\end{array}$} & \multicolumn{1}{c|}{ Definição } \\
\hline Risco & Probabilidade de ocorrência de perdas, danos ou lesões. \\
\hline Qualidade & Simplesmente a conformidade dos requisitos. \\
\hline Segurança & $\begin{array}{l}\text { Conservação da vida humana, sua efetividade e a prevenção de danos para itens de } \\
\text { acordo com os requisitos operacionais. }\end{array}$ \\
\hline Custo & $\begin{array}{l}\text { Recurso financeiro pago ou pagável pela aquisição de propriedade, materiais ou } \\
\text { serviços. }\end{array}$ \\
\hline Manutenção & $\begin{array}{l}\text { Medidas adequadas para manter um item, ou reparar/ restaurá-lo, a um estado } \\
\text { declarado, garantindo que os ativos físicos continuem cumprindo suas missões } \\
\text { declaradas. }\end{array}$ \\
\hline $\begin{array}{l}\text { Fatores } \\
\text { Humanos }\end{array}$ & $\begin{array}{l}\text { Garante que o projeto do equipamento, as tarefas humanas necessárias e o } \\
\text { ambiente de trabalho sejam compatíveis com os atributos sensoriais, perceptivos, } \\
\text { mentais e físicos do pessoal que o operará, manterá, controlará e apoiará. }\end{array}$ \\
\hline
\end{tabular}

Fonte: Omdahl (1988).

Neste trabalho estas áreas são denominadas de Áreas Associadas e são usadas no intuito de mapear todas as inter-relações do equipamento médico, de forma a aumentar sua confiabilidade.

\section{PROCESSO DE PROJETO DE EQUIPAMENTOS MÉDICOS (PPEM)}

De acordo com Back et al. (2008), o processo de desenvolvimento de produto é um conceito amplo que compreende os aspectos de planejamento e projeto, ao longo de todas as atividades da sequência do processo, desde a pesquisa de mercado, o projeto do produto, projeto do processo de fabricação, plano de distribuição e de manutenção até o descarte ou desativação do mesmo. Por esse conceito, entende-se desenvolvimento de produto como todo o processo de 
transformação de informações necessárias para a identificação da demanda, a produção e o uso do produto.

Para EM, o processo de desenvolvimento diferencia-se dos demais pelo contato direto dos equipamentos com os pacientes, tornando necessário que o produto seja confiável, robusto e preciso (OGRODNIK, 2012). Vários modelos voltados para o desenvolvimento de EM estão disponíveis na literatura como Pugh (1991), Das e Almonor (2000), Otto e Wood (2003), Pietzsch et al. (2009); El-Haik e Mekki (2011); Neelamkavil, Pardasani e Kernahan (2011); Ogrodnik (2012); Lantada (2013); Medina, Kremer e Wysk (2013); Pahl e Beitz (2013) e Ceretti et al. (2016).

A análise de tais modelos resultou em seis fases do desenvolvimento para EM: Projeto Informacional, Projeto Conceitual, Engenharia Básica, Projeto Detalhado, Produção e Vendas. A seguir, cada fase será explicada brevemente.

- Projeto Informacional: busca realizar análise de oportunidades do mercado, definição do problema, análise de concorrentes, identificação e análise das necessidades dos clientes que engloba as limitações/restrições e os fatores humanos/ usabilidade, análise competitiva, requisitos, especificações meta que compreende identificar problemas básicos. Ferramentas usualmente utilizadas nessa fase são: Questionários estruturados e semi-estruturados, listas de verificação, observação direta, brainstorming, Desdobramento da Função Qualidade (QFD) e Diagrama de Mudge (BACK et al, 2018).

- Projeto Conceitual: se destina a geração de soluções alternativas que atendam às especificações meta definidas na fase anterior, à avaliação e à seleção da melhor e mais inovadora concepção para o produto. A concepção selecionada representa $\mathrm{o}$ produto em suas principais funcionalidades $\mathrm{e}$ princípios de solução. Esta fase demanda criatividade, uma vez que se pressupõe a geração de soluções alternativas para aumentar a chance de sucesso de soluções inovadoras para o produto. Entre os métodos e ferramentas sugeridos para o projeto conceitual, cita-se: síntese de funções, TRIZ, matriz morfológica, brainstorming, analogias e métodos de seleção de soluções, como matriz multicritério (ALTSHULLER et al., 1989; CARVALHO, 1999; BACK et al., 2008 e PAHL e BEITZ, 2013). 
- Engenharia Básica: Também denominada de Projeto Preliminar nessa fase desenvolve-se o leiaute otimizado do produto no qual os materiais, as principais dimensões e processos de manufatura são definidos. $O$ resultado final é a determinação do leiaute final do produto, bem como sua viabilidade técnica e econômica. Nessa fase há maior intensidade de atividades de modelagem, simulação, análise, teste e otimização do produto, onde são aplicadas ferramentas como os sistemas CAD e CAE, construção de modelos e protótipos. Além disso, neste momento, toma-se a decisão por fazer ou comprar os componentes e/ou módulos do produto (BACK et al., 2008).

- Projeto Detalhado: destina-se a vários propósitos: aprovação do protótipo, finalização das especificações dos componentes, detalhamento do plano de manufatura e preparação da solicitação de investimento (BACK et al., 2008).

- Produção e Vendas. Com base nas fases já desenvolvidas e na documentação técnica, pode iniciar-se a produção e a colocação do produto no mercado. Fase em que o produto está em posse do cliente final, e são feitas manutenções assistenciais para garantir seu funcionamento até seu fim de vida, bem como são levantados dados de falha de tempo de vida. Tarefas principais: processo produtivo, logística de transporte, técnicas de vendas, instalação, instruções de uso, uso, manutenções, dados finais de falha.

Durante o PPEM, torna-se imprescindível o levantamento das legislações do país que norteiam o desenvolvimento de um EM. Para o desenvolvimento de equipamentos para saúde da Classe de Risco III e IV (classificado pela RDC $n^{\circ}$ 185/01) o qual inclui os equipamentos médicos de rádio frequência (RFA), foram relacionados instrumentos normativos que norteiam e delimitam o PPEM, chegandose a 31 legislações aplicáveis diretamente.

As legislações identificadas para esta classe de EM foram extraídas do repositório da Anvisa, membro do Fórum Regulatório Internacional de Dispositivos Médicos (International Medical Device Regulatory Forum, IMDRF) no Brasil e da ABNT (CERETTI et al., 2016; ABNT, 2017; ANVISA, 2017). São legislações aplicáveis a qualquer produto dentro dessas classes de risco, podendo ser que existam normas ou legislações adicionais para equipamentos específicos. 
O Quadro 2 apresenta uma síntese da fase do Projeto Informacional de um EM suas atividades, as áreas da confiabilidade envolvidas, os métodos e técnicas para análise de confiabilidade e a legislação associada.

Quadro 2 - Caracterização da confiabilidade segundo as fases do desenvolvimento e normas associadas

\begin{tabular}{|c|c|c|c|c|}
\hline $\begin{array}{c}\text { FASE DO } \\
\text { PPEM }\end{array}$ & ATIVIDADES & $\begin{array}{c}\text { ÁREAS } \\
\text { ASSOCIADAS }\end{array}$ & $\begin{array}{c}\text { MÉTODOS } \\
\text { E FERRAMENTAS }\end{array}$ & LEGISLAÇÕES \\
\hline $\begin{array}{c}\text { Projeto } \\
\text { Informa - } \\
\text { cional }\end{array}$ & $\begin{array}{c}\text { Análise de } \\
\text { oportunidade de } \\
\text { mercado; } \\
\text { Estratégias de } \\
\text { busca; } \\
\text { Ideias de produto; } \\
\text { definição do } \\
\text { problema de } \\
\text { projeto; } \\
\text { Identificação das } \\
\text { necessidades dos } \\
\text { clientes, } \\
\text { requisitos dos } \\
\text { clientes, } \\
\text { requisitos de } \\
\text { projeto, } \\
\text { especificações- } \\
\text { meta. }\end{array}$ & $\begin{array}{l}\text { Segurança, análise } \\
\text { de erros humanos }\end{array}$ & $\begin{array}{c}\text { Segurança (Análise } \\
\text { de risco premilinar, } \\
\text { análise de erro } \\
\text { humano, Análise de } \\
\text { Risco Operacional). } \\
\text { Análise de erros } \\
\text { humanos (Método da } \\
\text { Taxa de } \\
\text { Transmissão, Árvore } \\
\text { de Falhas) }\end{array}$ & $\begin{array}{c}\text { ABNT NBR IEC } \\
60601-1: 2010 \\
\text { ABNT NBR IEC } \\
60601-1-2: 2010 \\
\text { ABNT NBR IEC } \\
60601-1-6: 2011 \\
\text { ABNT NBR ISO } \\
13485: 2016 \\
\text { ABNT NBR ISO } \\
9001: 2015 \\
\text { RE } n^{\circ} 2605 \text { de } \\
11 / 08 / 2006 \\
\text { RE } n^{\circ} 3385 \text { de } \\
13 / 10 / 2006 \\
\text { RDC } n^{\circ} 14 \text { de } \\
05 / 04 / 2011 \\
\text { RDC } n^{\circ} 16 \text { de } \\
28 / 03 / 2013 \\
\text { RDC } n^{\circ} 22 \text { de } \\
15 / 03 / 2012 \\
\text { RDC } n^{\circ} 27 \text { de } \\
21 / 06 / 2011 \\
\text { RDC } n^{\circ} 27 \text { de } \\
15 / 05 / 2014 \\
\text { RDC } n^{\circ} 56 \text { de } \\
6 / 04 / 2011 \\
\text { RDC } n^{\circ} 156 \text { de } \\
11 / 08 / 2006 \\
\text { RDC } n^{\circ} 185 \text { de } \\
13 / 10 / 2006 \\
\text { IN no } 03 \text { de } 18 / 01 / 2010 \\
\text { IN no } 04 \text { de } 15 / 06 / 2012 \\
\text { IN no } 04 \text { de } 24 / 09 / 2015\end{array}$ \\
\hline
\end{tabular}

A seguir, será apresentado o estudo de caso presente artigo tem o objetivo de apresentar a fase de projeto informacional de um eletrodo de RF. 


\section{ESTUDO DE CASO}

\subsection{Eletrodo de Ablação por RadioFrequência}

O presente estudo de caso faz parte do projeto de pesquisa intitulado Software of Intensive Ablation (SOFIA) da Universidade de Brasília (UnB) cujo objetivo é desenvolver de um equipamento médico de RFA. Tal projeto é financiado pelo Ministério da Saúde em parceria com a UnB, sendo uma das frentes do Laboratório de Engenharia Biomédica (LaB), ambos vinculados ao Laboratório de Engenharia (LEI) e Inovação da Faculdade do Gama (FGA).

De forma mais detalhada, o equipamento de RFA a ser desenvolvido é constituído por um gerador de RFA, um eletrodo monopolar ativo e um software de controle. Dentro desse equipamento será estudado apenas o eletrodo que o compõe, um exemplo de eletrodo usado em tais procedimentos é exibido na Figura 1.

Figura 1 - Eletrodo de RFA modelo guarda chuva LeVeen 4.0 a) Vista periférica; b) Agulha de inserção c) Vista lateral e d) Dimensões do eletrodo
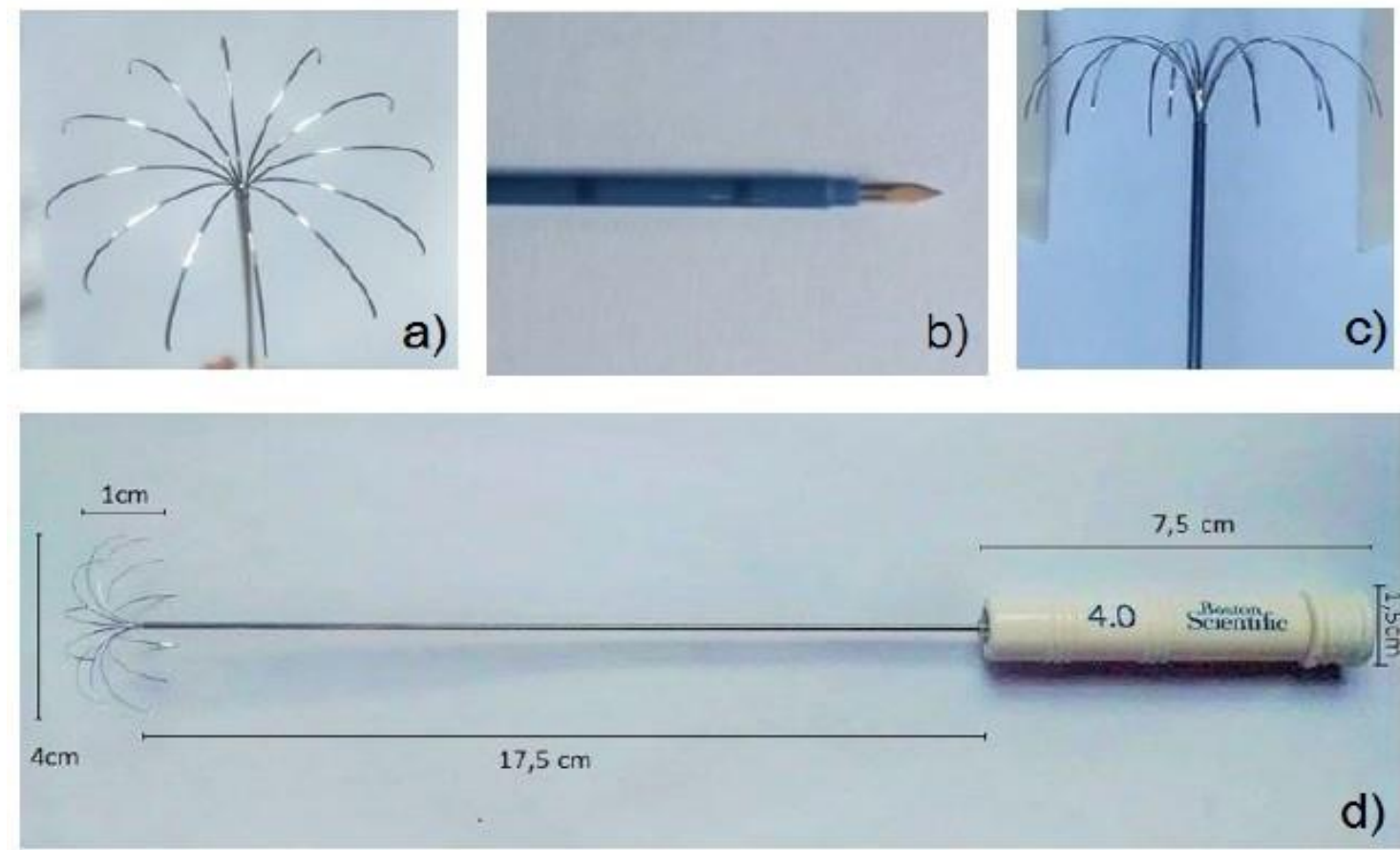

Fonte: Marques (2017).

O equipamento médico estudado é utilizado como forma de tratamento do Carcinoma Hepatocelular ( $\mathrm{CHC}$ ), que é um dos tumores malignos mais frequentes Revista Produção Online. Florianópolis, SC, v. 20, n. 4, p. 1238- 1259, 2020. 
no mundo sendo diagnosticado em mais de meio milhão de pessoas (GOMES et al., 2013). Este tipo histológico de câncer é agressivo, sendo classificado pela capacidade de se infiltrar dentro do órgão e comprometê-lo. Pode se apresentar na forma unifocal (único nódulo) ou nódulos multifocais, bem distribuídos e de tamanhos variados.

Segundo a Organização Mundial da Saúde (OMS) o CHC é a segunda causa de óbito por câncer. No Brasil, a incidência de CHC é considerada baixa, sendo maior incidente nos Estados do Espírito Santo e a Bahia (TIONG; MADDERN, 2011; BRASIL, 2013;). Dentre as formas de tratamento, são utilizados procedimentos e equipamentos para tentar diminuir ou até extinguir a região tumoral no fígado. Entre elas se encontra a ablação por RFA, que é um procedimento cirúrgico minimamente invasivo capaz de destruir lesões malignas nos pulmões, rins, glândulas suprarrenais, ósseas, baço, nódulos da mama, linfa, pélvis, próstata, tecido neural e fígado (IANNITTI et al., 2002; CARVALHO et al., 2011; RABELLO; SOUZA e JÚNIOR, 2014).

Nesse procedimento, o eletrodo, guiado por imagem, é inserido no tumor dentro do fígado e permite o tratamento de tumores de primeiro estágio. A passagem de corrente em alta frequência, tipicamente entre $400 \mathrm{kHz}$ a $500 \mathrm{kHz}$ gera calor no eletrodo e resulta no aumento da temperatura e consequentemente a queima das células cancerígenas (MARQUES, 2017).

Em uma operação de RFA a temperatura pode atingir até $1000^{\circ} \mathrm{C}$ na região próxima ao eletrodo. A morte celular entretanto, é considerada instantânea entre 50ㄷ e 60ำ (HAEMMERICH, 2010).

É considerado um dano irreversível quando o tumor necrosa devido a desnaturação da membrana da célula durante uma aplicação de RFA com duração de 2-5 min (CHEN; MIGA; JR, 2009). A lesão é iniciada do centro e o seu tamanho cresce associado com a condução térmica do tecido associado a um decaimento da impedância. Com a necrose do tecido essa impedância aumenta repentinamente, este evento é chamado de roll-off (CHEN; MIGA; JR, 2009; ALBA et al., 2011;; PENG; O'NEILL; PAYNE, 2011). Durante a necrose, restos de tecido ficam presos no eletrodo, inviabilizando seu uso para outras aplicações. O sucesso do procedimento da ablação está relacionado diretamente com o posicionamento 
correto do eletrodo, a sua geometria e a combinação ideal de potência e tempo aplicadas para garantir a necrose das células cancerígenas, evitando a morte dos tecidos sádios ao redor (MARQUES, 2017). As combinações de mais de um tipo de terapia para o tratamento do tumor, pode ser utilizada para atingir uma eficácia maior no tratamento de tumores. No tratamento do CHC, a RFA tem sido combinada com a infusão controlada de solução salina, a quimioterapia, a quimio ablação por etanol ou ácido acético e a embolização para aumentar a sua eficiência (LEE et al., 2004; GERMANI et al., 2010).

A seguir será apresentado a fase de projeto informacional de um eletrodo de ablação por radiofrequência (RFA).

\subsection{Projeto Informacional}

A fase do projeto informacional tem como objetivo identificar a informações de mercado em termos de produtos concorrentes e necessidades de clientes. Essas necessidades são convertidas em requisitos de clientes, depois requisitos de projetos e por fim, especificações meta do projeto.

\subsubsection{Atividade 1 - Identificação das Necessidades dos Clientes}

Dentro da fase de projeto informacional tem-se a atividade de identificação das necessidades dos clientes. As necessidades dos clientes identificadas foram obtidas com base em uma revisão das Normas da ABNT, Resoluções, Instruções Normativas da Anvisa. Utilizou-serem particular a ABNT NBR IEC 60601-2-2:2013 que trata dos requisitos particulares para a segurança básica e desempenho essencial de equipamentos cirúrgicos de alta frequência e acessórios cirúrgicos de alta frequência (ABNT, 02 de dezembro de 2013).

O Quadro 3 mostra as necessidades dos clientes de acordo com as fases do ciclo de vida de um EM e as áreas associadas à confiabilidade de dispositivos médicos mostradas no Quadro 1. É possível notar que ocorre maior concentração de necessidades de confiabilidade nos atributos de segurança, análise de risco e fatores humanos/ usabilidade e nas fases de engenharia básica e projeto detalhado. No total, foram levantadas 51 necessidades de clientes.

Revista Produção Online. Florianópolis, SC, v. 20, n. 4, p. 1238- 1259, 2020. 
De acordo com Otto e Wood (2003), é necessário priorizar as necessidades dos clientes, numerando-as em ordem de prioridade para tornar o projeto mais focado e enxuto possível. Entretanto, no caso de EM existem necessidades que devem ser obrigatoriamente satisfeitas por razões legislatórias, como é caso das necessidades relacionadas a confiabilidade. Por essa razão essas não serão priorizadas no presente artigo. 
Quadro 3 - necessidades dos clientes de acordo com as fases do ciclo de vida de um EM

(continua)

\begin{tabular}{|c|c|c|c|c|c|c|}
\hline 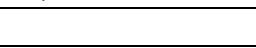 & \multicolumn{6}{|c|}{ ÁREAS ASSOCIADAS A CONFIABILIDADE } \\
\hline $\begin{array}{c}\text { FASES DO } \\
\text { CICLO DE VIDA }\end{array}$ & QUALIDADE & SEGURANÇA & ANÁLISE DE RISCO & MANUTENÇÃO & $\begin{array}{c}\text { FATORES HUMANOS/ } \\
\text { USABILIDADE }\end{array}$ & custos \\
\hline $\begin{array}{c}\text { Projeto } \\
\text { Informacional }\end{array}$ & & $\begin{array}{l}\text { - Os conectores ativos } \\
\text { do eletrodo que } \\
\text { possuam mais de um } \\
\text { pino devem ter um } \\
\text { espaçamento de pinos } \\
\text { fixo. }\end{array}$ & & & & \\
\hline $\begin{array}{l}\text { Projeto } \\
\text { Conceitual }\end{array}$ & & $\begin{array}{l}\text { - Ter poucas peças; } \\
\text { - Agrupamento de } \\
\text { peças. }\end{array}$ & & & & $\begin{array}{l}\text { - Ter durabilidade } \\
\text { superior ou igual ao } \\
\text { prazo de validade. }\end{array}$ \\
\hline $\begin{array}{l}\text { Engenharia } \\
\text { Básica }\end{array}$ & $\begin{array}{l}\text { - Aquisição de } \\
\text { componentes com } \\
\text { sólida reputação no } \\
\text { mercado; } \\
\text { - Cabos, encaixes e } \\
\text { conectores padrões } \\
\text { que facilitem a } \\
\text { montagem. }\end{array}$ & $\begin{array}{l}\text { - Partes elétricas dos } \\
\text { gatilhos do eletrodo } \\
\text { devem ser protegidas } \\
\text { para evitar a entrada de } \\
\text { líquidos; } \\
\text { - O circuito do paciente } \\
\text { deve ser isolado do terra } \\
\text { tanto para frequências } \\
\text { altas quanto baixas; } \\
\text {-Impedância do contato } \\
\text { elétrico entre a } \\
\text { superfície do local de } \\
\text { aplicação do eletrodo e } \\
\text { a conexão do cabo deve } \\
\text { ser pequena o suficiente } \\
\text { para evitar riscos de } \\
\text { queimadura no paciente; } \\
\text { - Documentos } \\
\text { acompanhantes de vem } \\
\text { instruir a inutilização do } \\
\text { eletrodo após a ativação } \\
\text { do dispositivo mecâ- } \\
\text { nico; } \\
\text {-Número comprimento } \\
\text { das hastes adequado. }\end{array}$ & $\begin{array}{l}\text { - Projetar o conector } \\
\text { elétrico de um } \\
\text { eletrodo removível } \\
\text { de forma a não entrar } \\
\text { em contato com o } \\
\text { corpo do paciente em } \\
\text { caso de des- conexão } \\
\text { inadvertida; } \\
\text { - O conector do } \\
\text { eletrodo que for } \\
\text { remoto deve ser } \\
\text { construído de forma a } \\
\text { não } \\
\text { entrar em contato com } \\
\text { as partes condutoras } \\
\text { das tomadas fixas de } \\
\text { rede ou dos } \\
\text { conectores de rede; } \\
\text {-lsolamento adequado } \\
\text { do cabo do eletrodo; } \\
\text { - Em caso de falha do } \\
\text { circuito do eletrodo, a } \\
\text { saída deve ser } \\
\text { desenergizada e } \\
\text { acionado um alarme } \\
\text { auditivo que não } \\
\text { pode ser ajustável } \\
\text { externamente. }\end{array}$ & $\begin{array}{l}\text { - Nas instruções de } \\
\text { utilização deve ter } \\
\text { um aviso para o } \\
\text { operador inspecionar } \\
\text { os acessórios, em } \\
\text { particular os } \\
\text { cabos dos } \\
\text { eletrodos; } \\
\text { - Visualização de fios } \\
\text { e conectores } \\
\text { facilitada para o } \\
\text { técnico. }\end{array}$ & $\begin{array}{l}\text { - Nas instruções de } \\
\text { utilização deve informar a } \\
\text { compatibilidade do } \\
\text { eletrodo com os outros } \\
\text { equipamen- tos; } \\
\text { - Formato de } \\
\text { manuseamento anatômico; } \\
\text { - Peso do equipamento de } \\
\text { forma a não cansar o } \\
\text { operador; } \\
\text { - Equipamento com } \\
\text { abertura ou desmonte } \\
\text { dificultada pelo usuário } \\
\text { (travas); } \\
\text { - Mecanismo de segurar; } \\
\text { - Forma que permita } \\
\text { segurar firmemente; } \\
\text { - Não escorregar da mão } \\
\text { do usuário; } \\
\text { - Fornecer orienta- ções ao } \\
\text { operador em relação à } \\
\text { con- veniência da } \\
\text { utilização de extra- ção } \\
\text { de fumaça-vapores; } \\
\text { - Número e } \\
\text { comprimento das hastes } \\
\text { adequado. }\end{array}$ & $\begin{array}{l}\text { - Ter poucas } \\
\text { peças; } \\
\text { - Agrupamento de } \\
\text { peças. }\end{array}$ \\
\hline
\end{tabular}

Revista Produção Online. Florianópolis, SC, v. 20, n. 4, p. 1238- 1259, 2020. 
Quadro 3 - necessidades dos clientes de acordo com as fases do ciclo de vida de um EM

(continuação)

\begin{tabular}{|c|c|c|c|c|c|c|}
\hline & \multicolumn{6}{|c|}{ ÁREAS ASSOCIADAS A CONFIABILIDADE } \\
\hline $\begin{array}{c}\text { FASES DO } \\
\text { CICLO DE VIDA }\end{array}$ & QUALIDADE & SEGURANÇA & $\begin{array}{l}\text { ANÁLISE DE } \\
\text { RISCO }\end{array}$ & MANUTENÇÃO & $\begin{array}{c}\text { FATORES HUMANOS/ } \\
\text { USABILIDADE }\end{array}$ & CUSTOS \\
\hline $\begin{array}{l}\text { Projeto } \\
\text { Detalhado }\end{array}$ & $\begin{array}{l}\text {-Tempo de queima } \\
\text { reduzido; } \\
\text { - Redução do tempo de } \\
\text { contato da cirurgia. }\end{array}$ & 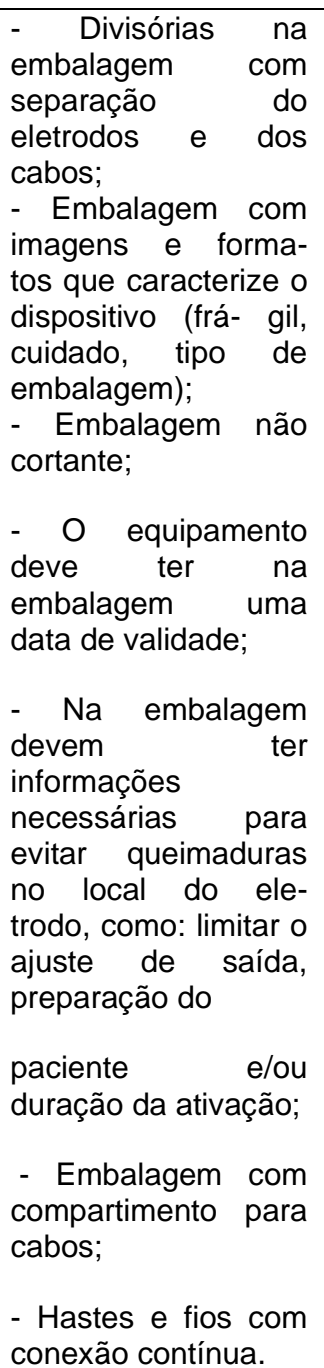 & $\begin{array}{l}\text { - Realização de } \\
\text { ensaios de segu- } \\
\text { rança elétrica e } \\
\text { compatibilidade } \\
\text { eletromagnética; } \\
\text { - Equipamento deve } \\
\text { ser marcado com } \\
\text { sinal de seguro ISO } \\
7010 \text {-W001; } \\
\text { - A marca deve ser } \\
\text { adjacente ao } \\
\text { dispositivo mecânico } \\
\text { ou localizado de } \\
\text { forma óbvia; } \\
\text { - Deve haver um } \\
\text { rótulo indicando o } \\
\text { peso máximo do } \\
\text { paciente em kg que } \\
\text { o equipamento deve } \\
\text { ser utilizado. }\end{array}$ & & $\begin{array}{l}\text { - Balanceamento da } \\
\text { vibração transmitida } \\
\text { pela mão do operador. }\end{array}$ & $\begin{array}{llr}\text { - Optar por travas de } & \text { der } \\
\text { baixo } & \text { custo; } & \\
- & \text { Aquisição de de } \\
\text { componentes com sólida } \\
\text { reputação no mercado. }\end{array}$ \\
\hline
\end{tabular}

Revista Produção Online. Florianópolis, SC, v. 20, n. 4, p. 1238- 1259, 2020. 
Quadro 3 - necessidades dos clientes de acordo com as fases do ciclo de vida de um EM

(conclusão)

\begin{tabular}{|c|c|c|c|c|c|c|}
\hline & \multicolumn{6}{|c|}{ ÁREAS ASSOCIADAS A CONFIABILIDADE } \\
\hline $\begin{array}{c}\text { FASES DO } \\
\text { CICLO DE VIDA }\end{array}$ & QUALIDADE & SEGURANÇA & $\begin{array}{l}\text { ANÁLISE DE } \\
\text { RISCO }\end{array}$ & MANUTENÇÃO & $\begin{array}{l}\text { FATORES HUMANOS/ } \\
\text { USABILIDADE }\end{array}$ & cUSTOS \\
\hline $\begin{array}{l}\text { Produção e } \\
\text { Vendas }\end{array}$ & $\begin{array}{l}\text { - Imagem guia com } \\
\text { diferenciação de tecidos. }\end{array}$ & \begin{tabular}{lr} 
- Equipamento de \\
uso único; \\
\multicolumn{3}{c}{ Proteção das } \\
- partes pontiagudas \\
ao descartar; \\
- Formato da \\
embalagem r que \\
facilite & a \\
armazenagem.
\end{tabular} & $\begin{array}{lr}\text { mecânico } & \text { Dispositivo } \\
\text { inutilizar } & \text { para } \\
\text { equipamento } & \text { após } \\
\text { uso; } & \\
-\quad \text { Material } & \text { da } \\
\text { embalagem } & \text { que } \\
\text { dificulte a entrada de } \\
\text { umidade. }\end{array}$ & $\begin{array}{l}\text { - Manutenção regular } \\
\text { dos cabos e eletrodo; } \\
\text { - Qualquer corrente } \\
\text { utilizada rara } \\
\text { monitorar para } \\
\text { continuidade elétrica do } \\
\text { cabo do eletrodo e } \\
\text { suas conexões deve } \\
\text { passar até uma seção } \\
\text { do eletrodo; - Garantia } \\
\text { de troca do } \\
\text { equipamento em casos } \\
\text { de defeito de } \\
\text { fabricação no eletrodo. }\end{array}$ & $\begin{array}{l}\text { - Durante o ciclo de } \\
\text { operação o des- canso } \\
\text { entre uma aplicação e } \\
\text { outra deve ser limitado. }\end{array}$ & $\begin{array}{l}\text { Imagem guia com } \\
\text { diferenciação de tecido }\end{array}$ \\
\hline
\end{tabular}

Revista Produção Online. Florianópolis, SC, v. 20, n. 4, p. 1238- 1259, 2020. 


\subsubsection{Atividade 2 - Estabelecimento dos Requisitos de Cliente}

O texto pode apresentar as citações diretas ou indiretas. Para o caso de citações diretas com até Definição dos requisitos de clientes que representa a necessidade levada à linguagem de projeto, de engenharia. No total as necessidades dos clientes foram desdobradas em 45 requisitos de cliente. A título de exemplo, o Quadro 4 mostra 10 requisitos de clientes.

Quadro 4 - Conversão das necessidades dos clientes em requisitos de cliente

\begin{tabular}{|c|c|c|c|}
\hline ITEM & $\begin{array}{l}\text { NECESSIDADES DOS } \\
\text { CLIENTES }\end{array}$ & OBSERVAÇÕES & $\begin{array}{l}\text { REQUISITOS DE } \\
\text { CLIENTE }\end{array}$ \\
\hline 1 & $\begin{array}{l}\text { Os conectores ativos } \\
\text { do eletrodo que } \\
\text { possuam mais de um } \\
\text { pino de- vem ter um } \\
\text { espaçamento de pinos } \\
\text { fixo. }\end{array}$ & $\begin{array}{l}\text { É preciso ter uma padronização no } \\
\text { espaçamento entre pinos, eletrodos } \\
\text { soltos estão proibidos. }\end{array}$ & $\begin{array}{l}\text { Ter espaçamento } \\
\text { de pinos nos } \\
\text { conectores fixos. }\end{array}$ \\
\hline 2 & $\begin{array}{l}\text { Nas instruções de } \\
\text { utilização deve } \\
\text { informar a } \\
\text { compatibilidade do } \\
\text { eletrodo com os outros } \\
\text { equipamentos. }\end{array}$ & $\begin{array}{l}\text { O eletrodo é conectado no gerador de } \\
\text { frequência, as instruções devem ser } \\
\text { claras em qual tipo ela é conectada e } \\
\text { quais equipamentos ocasi- onam } \\
\text { interferência }\end{array}$ & $\begin{array}{lr}\text { Informar } & \text { a } \\
\text { compatibilidade } & \\
\text { do eletrodo } & \text { com } \\
\text { outros } & \text { equi- } \\
\text { pamentos } & \text { nas } \\
\text { instruções } & \text { de } \\
\text { utilização. } & \\
\end{array}$ \\
\hline 3 & Ter poucas peças & $\begin{array}{l}\text { Quanto menor a quantidade de peças, } \\
\text { mais fácil e barata fica a montagem e } \\
\text { facilita a manutenção do equipamento. }\end{array}$ & Ter poucas peças \\
\hline 4 & Agrupamento de peças & $\begin{array}{l}\text { Agrupar o maior número possível de } \\
\text { peças. }\end{array}$ & \\
\hline 5 & $\begin{array}{l}\text { Ter durabilidade } \\
\text { superior ou igual } \\
\text { ao prazo de validade. }\end{array}$ & $\begin{array}{l}\text { Equipamentos descartáveis devem ter } \\
\text { prazo de validade, é preciso garantir que } \\
\text { o produto tenha durabilidade igual ou } \\
\text { superior ao seu prazo de validade. }\end{array}$ & $\begin{array}{l}\text { Ter durabilidade } \\
\text { superior ou igual ao } \\
\text { prazo de validade. }\end{array}$ \\
\hline 6 & $\begin{array}{l}\text { Cabos, encaixes e } \\
\text { conectores padrões } \\
\text { que facilitem a } \\
\text { montagem. }\end{array}$ & $\begin{array}{l}\text { Utilizar cabos, encaixes e conectores } \\
\text { com padrão IEEE, de forma a } \\
\text { interconectar os equipamentos e facilitar } \\
\text { sua montagem. }\end{array}$ & $\begin{array}{l}\text { Ter cabos, encaixes e } \\
\text { conectores padrões. }\end{array}$ \\
\hline 7 & $\begin{array}{l}\text { Aquisição de } \\
\text { componentes com } \\
\text { sólida reputação no } \\
\text { mercado. }\end{array}$ & $\begin{array}{l}\text { Componentes novos no mercado não } \\
\text { possuem um retorno das pessoas que o } \\
\text { utilizaram poucos dados de fa- lha, } \\
\text { acarretando riscos ao projeto. }\end{array}$ & $\begin{array}{l}\text { Usar componentes } \\
\text { com sólida reputação } \\
\text { no mercado. }\end{array}$ \\
\hline 8 & $\begin{array}{l}\text { Partes elétricas dos } \\
\text { gatilhos do eletrodo } \\
\text { devem ser protegidas } \\
\text { para evitar a entrada } \\
\text { de líquidos. }\end{array}$ & $\begin{array}{l}\text { No momento da cirurgia o eletrodo entra } \\
\text { em contato com líquidos corporais que } \\
\text { podem danificar seu circuito. }\end{array}$ & $\begin{array}{l}\text { Proteger as partes } \\
\text { elétricas dos gatilhos. }\end{array}$ \\
\hline 9 & $\begin{array}{l}\text { O circuito do paciente } \\
\text { deve ser isolado do } \\
\text { terra tanto para } \\
\text { frequências } \quad \text { altas } \\
\text { quanto baixas. }\end{array}$ & $\begin{array}{l}\text { O eletrodo deve ser isolado do terra em } \\
\text { altas frequências para fornecer uma } \\
\text { referência estável de tensão aos sinais e } \\
\text { circuitos. }\end{array}$ & $\begin{array}{l}\text { Isolar o circuito do } \\
\text { paciente para altas e } \\
\text { baixas frequências. }\end{array}$ \\
\hline
\end{tabular}


Quadro 4 - Conversão das necessidades dos clientes em requisitos de cliente

\begin{tabular}{|c|c|c|c|}
\hline ITEM & $\begin{array}{c}\text { NECESSIDADES DOS } \\
\text { CLIENTES }\end{array}$ & OBSERVAÇÕES & $\begin{array}{l}\text { REQUISITOS DE } \\
\text { CLIENTE }\end{array}$ \\
\hline 10 & $\begin{array}{l}\text { Impedância do } \\
\text { contato elétrico entre } \\
\text { a superfície do local } \\
\text { de aplica- ção do } \\
\text { eletrodo e a conexão } \\
\text { do cabo deve ser } \\
\text { pequena o suficiente } \\
\text { para } \\
\text { evitar riscos. }\end{array}$ & $\begin{array}{l}\text { Para evitar riscos de quei- madura do } \\
\text { paciente devido ao aquecimento } \\
\text { ôhmico du- rante a passagem da } \\
\text { corrente cirúrgica de AF. }\end{array}$ & $\begin{array}{l}\text { Ter impedância } \\
\text { pequena su- ficiente } \\
\text { para evitar riscos de } \\
\text { queimadura. }\end{array}$ \\
\hline
\end{tabular}

\subsubsection{Atividade 3 - Estabelecimento dos Requisitos de Projeto}

Os requisitos de cliente foram desdobrados em requisitos de projeto, ou sejam em requisitos técnicos em termos de números, tolerâncias, indicações qualitativas que posteriormente serão transformados em especificações meta do PPEM. O desdobramento é apresentado no Quadro 5, onde é mostrado, resumidamente os requisitos do produto.

Quadro 5 - Desdobramento dos requisitos de clientes em requisitos de projeto.

\begin{tabular}{|c|c|c|}
\hline ITEM & REQUISITOS DE CLIENTE & REQUISITOS DE PROJETO \\
\hline 1 & $\begin{array}{l}\text { Ter espaçamento de pinos nos } \\
\text { conectores fixos. }\end{array}$ & Distância entre conectores (cm) \\
\hline 2 & $\begin{array}{l}\text { Informar a compatibilidade do eletrodo } \\
\text { com outros equipamentos nas } \\
\text { instruções de utilização. }\end{array}$ & $\begin{array}{l}\text { Quantidade de instruções no manual } \\
\text { (adimensional) }\end{array}$ \\
\hline 3 & Ter poucas peças. & Quantidade de peças (adimensional) \\
\hline 4 & $\begin{array}{l}\text { Ter durabilidade superior ou igual ao } \\
\text { prazo de validade. }\end{array}$ & Prazo de validade (anos) \\
\hline 5 & $\begin{array}{l}\text { Ter cabos, encaixes e conectores } \\
\text { padrões. }\end{array}$ & Tempo dos fabricantes no mercado (anos) \\
\hline 6 & $\begin{array}{l}\text { Usar componentes com sólida reputação } \\
\text { no mercado. }\end{array}$ & Tempo dos fabricantes no mercado (anos) \\
\hline 7 & Proteger as partes elétricas dos gatilhos. & $\begin{array}{l}\text { Dimensões do equipamento (Comprimento } \\
\text { da empunhadura (cm); Largura da } \\
\text { empunhadura (cm); Diâmetro do eletrodo } \\
\text { aberto }(\mathrm{cm}) \text {; Comprimento da cânula }(\mathrm{cm}))\end{array}$ \\
\hline 8 & $\begin{array}{l}\text { Isolar o circuito do paciente para altas e } \\
\text { baixas frequências. }\end{array}$ & Frequência de corrente de fuga AF (kHz) \\
\hline 9 & $\begin{array}{l}\text { Ter impedância pequena suficiente para } \\
\text { evitar riscos de queimadura. }\end{array}$ & Impedância $(\Omega)$ \\
\hline 10 & $\begin{array}{l}\text { Instruir a não utilização do eletrodo após } \\
\text { a ativação do dispositivo mecânico. }\end{array}$ & $\begin{array}{l}\text { Quantidade de instruções no manual } \\
\text { (adimensional) }\end{array}$ \\
\hline
\end{tabular}




\subsubsection{Atividade 3 - Especificações Meta}

Por último, são definidas as especificações do projeto, que são critérios mensuráveis que o produto deve ser designado a satisfazer. Elas devem ser metas mensuráveis para a equipe de projeto, serem estabelecidas inicialmente no PPEM e atualizadas com frequência (OTTO; WOOD, 2003). Cada requisito de produto constitui uma meta do projeto, portanto, foram levantadas 34 especificações meta. As especificações meta do projeto analisado, são dispostas de forma sintetizada, no Quadro 6.

Quadro 6 - Especificações meta do projeto analisado

(continua)

\begin{tabular}{|c|c|c|c|c|c|}
\hline ITEM & $\begin{array}{l}\text { ESPECIFICA- } \\
\text { ÇÃO }\end{array}$ & META & SENSOR & $\begin{array}{c}\text { SAÍDAS } \\
\text { INDESEJADAS }\end{array}$ & RESTRIÇÕES \\
\hline 1 & $\begin{array}{l}\text { Distância entre } \\
\text { conectores }(\mathrm{cm})\end{array}$ & $\begin{array}{l}\text { Espaçament } \\
\text { o de pinos } \\
\text { fixo }\end{array}$ & Inspeção & $\begin{array}{l}\text { Despadronização } \\
\text { e interferência } \\
\text { entre as conexões }\end{array}$ & $\begin{array}{l}\text { Os conectores } \\
\text { que possuam } \\
\text { mais de um pino } \\
\text { devem ter um } \\
\text { espaçamento } \\
\text { de pinos fixo. }\end{array}$ \\
\hline 2 & $\begin{array}{l}\text { Quantidade de } \\
\text { instruções no } \\
\text { manual } \\
\text { (adimensional) }\end{array}$ & $\begin{array}{l}\text { Aberto ao } \\
\mathrm{s} \\
\text { desenvolvedo } \\
\text { res }\end{array}$ & $\begin{array}{l}\text { Inspeção } \\
\text { visual }\end{array}$ & $\begin{array}{l}\text { Má utilização } \\
\text { equipamento }\end{array}$ & $\begin{array}{l}\text { A quantidade de } \\
\text { instruções pode } \\
\text { se mo- dificar ao } \\
\text { longo do projeto. }\end{array}$ \\
\hline 3 & $\begin{array}{l}\text { Quantidade de } \\
\text { peças } \\
\text { (adimensional) }\end{array}$ & $<10$ & $\begin{array}{l}\text { Inspeção } \\
\text { visual }\end{array}$ & Falhas nas peças & $\begin{array}{l}\text { Quanto maior } \\
\text { a quantidade de } \\
\text { peças, maiores } \\
\text { são as chan- ces } \\
\text { de haver falhas } \\
\text { nas peças do } \\
\text { produto. }\end{array}$ \\
\hline 4 & $\begin{array}{l}\text { Prazo de validade } \\
\text { (anos) }\end{array}$ & $\begin{array}{l}\text { Aberto ao } \\
\mathrm{s} \\
\text { desenvolvedo- } \\
\text { re }\end{array}$ & $\begin{array}{l}\text { Inspeção } \\
\text { do manual }\end{array}$ & $\begin{array}{l}\text { Desgaste } \\
\text { antecipado à } \\
\text { validade }\end{array}$ & $\begin{array}{l}\text { Para } \\
\text { equipamentos } \\
\text { com potência } \\
\text { superior a } 50 \mathrm{~W} \\
\text { que necessitam } \\
\text { de um eletrodo } \\
\text { neutro de uso } \\
\text { único. }\end{array}$ \\
\hline 5 & $\begin{array}{l}\text { Tempo dos } \\
\text { fabricantes no } \\
\text { mercado(anos) }\end{array}$ & $\geq 5$ & $\begin{array}{l}\text { Histórico } \\
\text { dos } \\
\text { fabricantes }\end{array}$ & $\begin{array}{l}\text { Algum componente } \\
\text { quebrar ou se com- } \\
\text { portar de maneira } \\
\text { inesperada durante } \\
\text { o uso do aparelho }\end{array}$ & \\
\hline 6 & $\begin{array}{l}\text { Comprimento da } \\
\text { punhadura }(\mathrm{cm})\end{array}$ & $5-9$ & $\begin{array}{l}\text { Trena para } \\
\text { medidas }\end{array}$ & Erro de medida & Inexistente \\
\hline
\end{tabular}

Revista Produção Online. Florianópolis, SC, v. 20, n. 4, p. 1238- 1259, 2020. 
Quadro 6 - Especificações meta do projeto analisado

\begin{tabular}{|l|l|l|l|l|l|}
\hline ITEM & \multicolumn{1}{|c|}{$\begin{array}{c}\text { ESPECIFICA- } \\
\text { ÇÃo }\end{array}$} & META & SENSOR & \multicolumn{1}{|c|}{$\begin{array}{c}\text { SAÍDAS } \\
\text { INDESEJADAS }\end{array}$} & RESTRIÇÕES \\
\hline 7 & $\begin{array}{l}\text { Largura da } \\
\text { empunhadura } \\
(\mathrm{cm})\end{array}$ & $1-3$ & $\begin{array}{l}\text { Trena para } \\
\text { medidas }\end{array}$ & Erro de medida & Inexistente \\
\hline 8 & $\begin{array}{l}\text { Diâmetro do } \\
\text { eletrodo aberto } \\
(\mathrm{cm})\end{array}$ & $3-5$ & Paquímetro & Erro de medida & Inexistente \\
\hline 9 & $\begin{array}{l}\text { Comprimento da } \\
\text { cânula (cm) }\end{array}$ & $12-25$ & $\begin{array}{l}\text { Trena para } \\
\text { medidas }\end{array}$ & $\begin{array}{l}\text { De acordo com } \\
\text { equi pamento } \\
\text { similar }\end{array}$ \\
\hline 10 & $\begin{array}{l}\text { Frequência de } \\
\text { corrente de fuga } \\
\text { AF }(\mathrm{kHz})\end{array}$ & $300-1000$ & & $\begin{array}{l}\text { Não isolamento do } \\
\text { circuito do paciente } \\
\text { para altas e baixas } \\
\text { frequências. }\end{array}$ & \\
\hline
\end{tabular}

\section{CONSIDERAÇÕES}

O desenvolvimento de EM demanda atenção especial pela relação direta com a saúde e vida dos pacientes. Seu desenvolvimento deve ser robusto, eficaz, seguro e confiável, sendo pressionado pelas exigências dos órgãos da saúde quanto ao atendimento a aspectos legais. Levando em consideração essas características, o presente artigo buscou apresentar o projeto informacional de um eletrodo de radiofrequência que faz parte do Software of Intensive Ablation (SOFIA) da UnB.

A aplicação de uma metodologia de projeto sistemática possibilitou definir uma sequência de etapas e atividades de fundamental importância para atingir o objetivo proposto. O projeto informacional apresentado é uma fase primordial para o processo de desenvolvimento do EM em questão, pois as especificações-meta estabelecidas nessa fase irão guiar as fases seguintes do projeto. Sugere-se a execução de testes no EM para que se possa fazer uma análise, por exemplo da confiabilidade, natureza das falhas e disponibilidade.

\section{REFERÊNCIAS}

ASSOCIAÇÃO BRASILEIRA DE NORMAS TÉCNICAS. ABNT NBR 5462:1994 Confiabilidade e mantenabilidade. Rio de Janeiro: ABNT, 1994.

ASSOCIAÇÃO BRASILEIRA DE NORMAS TÉCNICAS. ABNT NBR IEC 60601-2-2 Equipamento eletromédico parte 2-2: Requisitos particulares para a segurança básica e desem- penho essencial de equipamentos cirúrgicos de alta frequência e acessórios cirúrgicos de alta frequência. Rio de Janeiro: ABNT, 2013. 
ASSOCIAÇÃO BRASILEIRA DE NORMAS TÉCNICAS. Catálogo de normas produtos para saúde. 2017. Disponível em: http://www.abnt.org.br/normalizazao/abnt-catalogo . Acesso em: 07 abr. 2017.

ASSOCIAÇÃO BRASILEIRA DE NORMAS TÉCNICAS. ABNT NBR IEC 60601-1-6: Versão Corrigida:2013. Equipamento eletromédico parte 1-6: Requisitos gerais para segurança básica e desempenho essencial - norma colateral: Usabilidade. Rio de Janeiro: ABNT, 2011.

ASSOCIAÇÃO BRASILEIRA DE NORMAS TÉCNICAS. ABNT NBR IEC 60601-1. Equipamento eletromédico parte 1: Requisitos gerais para segurança básica e desempenho essencial. Rio de Janeiro: ABNT, 2010. Válida a partir de: 01 de janeiro de 2012.

ASSOCIAÇÃO BRASILEIRA DE NORMAS TÉCNICAS. ABNT NBR IEC 60601-1-2 Errata 1:2013. Equipamento eletromédico parte 1-2: Requisitos gerais para segurança básica e desempenho essencial - norma colateral: Compatibilidade eletromagnética requisitos e ensaio. Rio de Janeiro: ABNT, 2013.

ASSOCIAÇÃO BRASILEIRA DE NORMAS TÉCNICAS. ABNT NBR ISO 9001 - Sistemas de gestão da qualidade - requisitos. Rio de Janeiro: ABNT, 2015. Válida a partir de: 30 de outubro de 2015.

ASSOCIAÇÃO BRASILEIRA DE NORMAS TÉCNICAS. ABNT NBR ISO 13485 Produtos para saúde - sistemas de gestão da qualidade — requisitos para fins regulamentares. Rio de Janeiro: ABNT, 2016.

ALBA, J. et al. Theoretical and experimental study on rf tumor ablation with internally cooled electrodes: when does the roll-off occur? In: IEEE. Engineering in Medicine and Biology Society, EMBC, 2011 Annual International Conference of the IEEE. [S.I.], p. 314-317, 2011. https://doi.org/10.1109/IEMBS.2011.6090082

ANVISA. Agência Nacional de Vigilância Sanitária. Instrução Normativa - IN no. 21. Dispõe sobre os procedimentos de inspeção em boas práticas clínicas para ensaios clínicos com dispositivos médicos em investigação. [S.I.], 02 de outubro de 2017. DOU, n. 190, Seção 1, p.47, 03 de outubro de 2017.

ANVISA. Agência Nacional de Vigilância Sanitária. Resolução de Diretoria Colegiada RDC no. 14 - Institui o regulamento técnico com os requisitos para agrupamento de materiais de uso em saúde para fins de registro e cadastro na anvisa e adota etiquetas de rastreabilidade para produtos implantáveis. [S.I.], 05 de abril de 2011. DOU, n. 69, Seção 1, p. 60, 11 de abril de 2011.

ANVISA. Agência Nacional de Vigilância Sanitária. Resolução de Diretoria Colegiada - RDC no. 56. Os produtos para a saúde devem atender aos requisitos essenciais de segurança e eficácia aplicáveis a estes produtos, referidos no regulamento técnico anexo a esta resolução. [S.I.], 06 de abril de 2001. DOU, n. 70, Seção 1, p. 28, 10 de abril de 2001.

ANVISA. Agência Nacional de Vigilância Sanitária. Resolução de Diretoria Colegiada $R D C$ no. 156. Dispõe sobre o registro, rotulagem e reprocessamento de produtos médicos, e dá outras providências. [S.I.], 11 de agosto de 2006. DOU, n. 155, Seção 1, p. 25, 14 de agosto de 2006.

ANVISA. Agência Nacional de Vigilância Sanitária. Resolução de Diretoria Colegiada RE no. 2605. Reprocessamento de produtos para a saúde. [S.I.], 11 de agosto de 2006. DOU, n.155, Seção 1, p. 28, 14 de agosto de 2006. 
ANVISA. Agência Nacional de Vigilância Sanitária. Resolução de Diretoria Colegiada RE no. 3385. Lista de produtos para saúde cujo relatório de informações econômicas deverá ser encaminhado ao núcleo de assessoramento econômico em regulação. [S.I.], 13 de outubro de 2006. DOU, n. 198, Seção 1, 16 de outubro de 2006.

ANVISA. Agência Nacional de Vigilância Sanitária. Resolução de Diretoria Colegiada RDC no. 27. Institui o protocolo eletrônico para emissão de certificado de produto (certificado de cadastro ou registro de produto para a saúde) e certidão para governo estrangeiro (certidão de cadastro ou registro para exportação de produto para saúde). [S.I.], 15 de maio de 2013. DOU, n. 94, Seção 1, p. 145, 17 de maio de 2013.

ANVISA. Agência Nacional de Vigilância Sanitária. Instrução Normativa - IN no. 03. Estabelece e divulga definições adotadas na resolução rdc no. 185, de 13 de outubro de 2006. [S.I.], 18 de janeiro de 2010. DOU, n. 14, Seção 1, p. 45, 21 de janeiro de 2010.

ANVISA. Agência Nacional de Vigilância Sanitária. Resolução de Diretoria Colegiada RDC no. 27. Dispõe sobre os procedimentos para certificação compulsória dos equipamentos sob regime de vigilância sanitâria. [S.I.], 21 de junho de 2011. DOU, n. 119, Seção 1, p. 86, 22 de junho de 2011.

ANVISA. Agência Nacional de Vigilância Sanitária. Resolução de Diretoria Colegiada - RDC no. 22. Aprova os procedimentos comuns para as inspeções nos fabricantes de produtos médicos e produtos para diagnóstico de uso in vitro nos estados partes, e dá outras providências. [S.I.], 23 de abril de 2013. DOU, n. 78, Seção 1, p. 51, 24 de abril de 2013.

ANVISA. Agência Nacional de Vigilância Sanitária. Instrução normativa - in nำ 49, de 22 de novembro de 2019 - Aprova a lista de normas técnicas, cujos parâmetros devem ser adotados para a certificação de conformidade, no âmbito do sistema brasileiro de avaliação da conformidade (sbac). [S.I.], 24 de setembro de 2015. DOU, n. 184, Seção 1, p. 72, 25 de setembro de 2015.

ANVISA. Agência Nacional de Vigilância Sanitária. Resolução de Diretoria Colegiada $R D C$ no. 16. Aprova o regulamento técnico de boas práticas de fabricação de produtos médicos e produtos para diagnóstico de uso in vitro e dá outras providências. [S.I.], 28 de março de 2013. DOU, n. 61, Seção 1, p. 75, 01 de abril de 2013.

BACK, N. et al. Projeto integrado de produtos: planejamento, concepção e modelagem. Barueri: Malone, 2008.

BRASIL, M. da Saúde de. Diretrizes metodológicas: elaboração de estudos para avaliação de equipamentos médico-assistenciais. [S.I.]: Secretaria de Ciência, Tecnologia e Insumos Estratégicos. Departamento de Ciência e Tecnologia, 2013.

CARVALHO, G. F. de et al. Avaliação dos efeitos da radiofrequência no tecido conjuntivo. Revista Brasileira de Medicina, v. 3, p. 10-25, 2011.

CERETTI, E. et al. Biomedical devices: design, prototyping, and manufacturing. [S.I.]: John Wiley \& Sons, 2016.

CHEN, C.-C. R.; MIGA, M. I.; JR, R. L. G. Optimizing electrode placement using finiteelement models in radiofrequency ablation treatment planning. IEEE transactions on biomedical engineering, IEEE, v. 56, n. 2, p. 237-245, 2009. https://doi.org/10.1109/TBME.2008.2010383 
DAS, S. K.; ALMONOR, J. B. A concurrent engineering approach for the development of medical devices. International Journal of Computer Integrated Manufacturing, v. 13, n. 2, p. 139-147, 2000. https://doi.org/10.1080/095119200129984

DHILLON, B. S. Medical device reliability and associated areas. [S.I.]: CRC Press, 2000. https://doi.org/10.1201/9781420042238

EL-HAIK, B.; MEKKI, K. S. Medical device design for six sigma: a road map for safety and effectiveness. [S.I.]: John Wiley \& Sons, 2011.

GERMANI, G. et al. Clinical outcomes of radiofrequency ablation, percutaneous alcohol and acetic acid injection for hepatocelullar carcinoma: a meta-analysis. Journal of hepatology, v. 52, n. 3, p. 380-388, 2010. https://doi.org/10.1016/j.jhep.2009.12.004

GOMES, M. A. et al. Hepatocellular carcinoma: epidemiology, biology, diagnosis, and therapies. Revista da Associação Médica Brasileira, v. 59, n. 5, p. 514-524, 2013. https://doi.org/10.1016/S2255-4823(13)70511-3

HAEMMERICH, D. Biophysics of radiofrequency ablation. Critical Reviews ${ }^{\text {TM }}$ in Biomedical Engineering, v. 38, n. 1, 2010.

https://doi.org/10.1615/CritRevBiomedEng.v38.i1.50

HEGDE, V.; RAHEJA, D. Design for reliability in medical devices. In: IEEE. Reliability and Maintainability Symposium (RAMS), 2010 Proceedings-Annual. [S.I.], 2010. p. 16. https://doi.org/10.1109/RAMS.2010.5448077

IANNITTI, D. A. et al. Hepatic radiofrequency ablation. Archives of Surgery, American Medical Association, v. 137, n. 4, p. 422-427, 2002.

https://doi.org/10.1001/archsurg.137.4.422

KING, P. H.; FRIES, R. C.; JOHNSON, A. T. Design of biomedical devices and systems. [S.I.]: CRC Press, 2014.

LANTADA, A. D. Handbook on advanced design and manufacturing technologies for bio- medical devices. [S.I.]: Springer, 2013. https://doi.org/10.1007/978-1-4614-6789-2

LEE, J. M. et al. Combined radiofrequency ablation and acetic acid hypertonic saline solution instillation: an in vivo study of rabbit liver. Korean journal of radiology, v. $5, n$. 1, p. 31-38, 2004. https://doi.org/10.3348/kjr.2004.5.1.31

LIN, Q.-L. et al. Human reliability assessment for medical devices based on failure mode and effects analysis and fuzzy linguistic theory. Safety science, v. 62, p. 248-256, 2014.

https://doi.org/10.1016/j.ssci.2013.08.022

MARQUES, M. P. Desenvolvimento de um eletrodo expansível de níquel-titânio para ablação hepática por radiofrequência. 2017.

MARTINI, F. P. Práticas de manutenção em equipamentos de diagnóstico e terapia em serviços de oftalmologia na cidade de Porto Alegre. 2005.

MEDINA, L. A.; KREMER, G. E. O.; WYSK, R. A. Supporting medical device development: a standard product design process model. Journal of Engineering Design, v. 24, n. 2, p. 83-119, 2013. https://doi.org/10.1080/09544828.2012.676635 
NEELAMKAVIL, J.; PARDASANI, A.; KERNAHAN, M. Traceability in medical devices design \& manufacturing. Proceedings of the Canadian Engineering Education Association, 2011. https://doi.org/10.24908/pceea.v0i0.3968

OGRODNIK, P. J. Medical device design: innovation from concept to market. [S.I.]: Academic Press, 2012.

OMDAHL, T. P. Reliability, availability, and maintainability: RAM: dictionary. [S.I.]: American Society for Quality Control, 1988.

OTTO, K. N.; WOOD, K. L. Product design: techniques in reverse engineering and new product development. [S.I.]: Prentice Hall, 2003.

PAHL, G.; BEITZ, W. Engineering design: a systematic approach. [S.I.]: Springer Science \& Business Media, 2013.

PENG, T.; O'NEILL, D.; PAYNE, S. A two-equation coupled system for determination of liver tissue temperature during thermal ablation. International Journal of Heat and Mass Transfer, v. 54, n. 9-10, p. 2100-2109, 2011. https://doi.org/10.1016/j.ijheatmasstransfer.2010.12.019

PIETZSCH, J. B. et al. Stage-gate process for the development of medical devices. Journal of Medical Devices, American Society of Mechanical Engineers, v. 3, n. 2, p. 021004 , 2009. https://doi.org/10.1115/1.3148836

PUGH, S. Total design: integrated methods for successful product engineering. 1990, 1991.

RABELLO, F. B.; SOUZA, C. D.; JÚNIOR, J. A. F. Update on hypertrophic scar treatment. Clinics, v. 69, n. 8, p. 565-573, 2014. https://doi.org/10.6061/clinics/2014(08)11

RAHEJA, D. G.; GULLO, L. J. Design for reliability. [S.I.]: John Wiley \& Sons, 2012. https://doi.org/10.1002/9781118310052

ROMERO, M.; CORPART, G. 5 mega trends in latin american heathcare: A look at major shifts in 2018 and for decades to come. In: Global Health Intelligence. [S.I.: s.n.], 2018.

TIONG, L.; MADDERN, G. Systematic review and meta-analysis of survival and disease recurrence after radiofrequency ablation for hepatocellular carcinoma. British Journal of Surgery, v. 98, n. 9, p. 1210-1224, 2011. https://doi.org/10.1002/bjs.7669

WOO, S. Reliability design of mechanical systems: a guide for mechanical and civil engineers. [S.I.]: Springer, 2017. https://doi.org/10.1007/978-3-319-50829-0

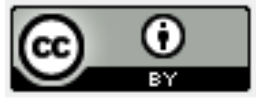

Artigo recebido em: 20/05/2020 e aceito para publicação em: 05/12/2020

DOI: http://dx.doi.org/10.14488/1676-1901. v20i4.4057 